\title{
Australia-Modified Karnofsky Performance Status 20
}

National Cancer Institute

\section{Source}

National Cancer Institute. Australia-Modified Karnofsky Performance Status 20. NCI

Thesaurus. Code C107492.

Totally bedfast and requiring extensive nursing care by professionals and/or family. 